\title{
Custom-Made Endograft for Endovascular Repair of Thoraco- Abdominal Aneurysm and Type B Dissection: Single-Centre Experience
}

\author{
Pierleone Lucatelli ${ }^{1,2}$ (D) Marco Cini ${ }^{1}$ - Antonio Benvenuti ${ }^{3}$ Luca Saba ${ }^{4}$. \\ Giulio Tommasino ${ }^{3}$ Giulia Guaccio ${ }^{3}$ - Graham Munneke ${ }^{5}$ Eugenio Neri $^{3}$. \\ Carmelo Ricci ${ }^{1}$
}

Received: 1 February 2018/ Accepted: 23 April 2018

(C) Springer Science+Business Media, LLC, part of Springer Nature and the Cardiovascular and Interventional Radiological Society of Europe (CIRSE) 2018

\begin{abstract}
Aims To report a series of patients treated with the Jotec custom-made endograft for thoraco-abdominal aneurysms and dissections and identify predictive factors for reintervention.

Methods We retrospectively analysed 49 patients unsuitable for surgery, treated between 2011 and 2017 (71.3 \pm 9.5 years; 15 females). Indications included Crawford type 4 aneurysm in 25 patients, type 3 in 13, type 2 in 4, type 1 in 2 and chronic aneurysmal dilatation of the false lumen following dissection in 5 cases. Mean aneurysm diameter was $58.7 \pm 8.4 \mathrm{~mm}$. The study aims were to assess procedural success, complications rate, mortality
\end{abstract}

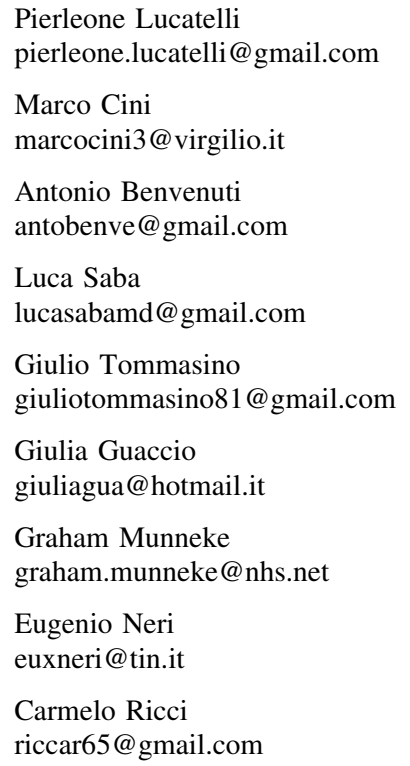

and long-term follow-up. We also analysed factors that predicted the need for re-intervention.

Results The endograft was successfully deployed in all patients, catheterization of the fenestration and/or branches was achieved in 152/156 (97.4\%) vessels. Early complications occurred in 10 patients ( 3 paraplegia, 3 haemorrhages, pancreatitis, aortic rupture, iliac artery rupture, 2 strokes). Thirty-day mortality was $10.2 \%$ and 180-day mortality $14.3 \%$; two non procedure related deaths occurred. Mean follow-up was $23.6 \pm 29.9$ months [range 1-80]. No patients needed surgical explantation or developed significant renal impairment. Endoleak rate was $34.6 \%$ and re-intervention rate $9.7 \%$. The aneurysm sac reduced or was stable in 36/49, and enlarged in $9 / 49$

1 Vascular and Interventional Radiology Unit, Azienda Ospedaliera Universitaria Senese, Viale Mario Bracci, 16, 53100 Siena, Italy

2 Vascular and Interventional Radiology Unit, Sapienza University of Rome, Viale Regina Elena, 324, 00161 Rome, Italy

3 Cardiac and Great Vessels Surgery Unit, University of "Siena", Viale Mario Bracci, 16, 53100 Siena, Italy

4 Department of Radiology, Azienda Ospedaliero Universitaria (AOU), di Cagliari - Polo di Monserrato s.s. 554, 09045 Monserrato (Cagliari), Italy

5 University College, London Hospital, London, UK 
patients prompting re-intervention. Primary, primary-assisted and secondary patency of fenestrations/branches at 80 months was 90,96 and $100 \%$. Re-intervention was required more frequently in braches than in fenestrations, most commonly the external type branches.

Conclusions The results of the Jotec endograft are comparable to other devices, with acceptable complication and re-intervention rates. Fenestration and inner-branch should be preferred due to lower re-intervention rates.

Keywords b-EVAR - f-EVAR - TEVAR - EVAR . Thoraco-abdominal aneurysm - Type B dissection . Endovascular repair

\section{Introduction}

The management of thoraco-abdominal aneurysms and false lumen aneurysmal dilatation in type B chronic dissection patients still represents a major challenge, due to their high risk of spontaneous rupture and death [1].

During the last decade there has been a shift in treatment modality moving from open surgical reconstruction in favour of the less invasive endovascular repair. Fenestrated or multi-branch devices have been reported to potentially reduce both morbidity and mortality [2]. Two different types of endovascular devices are currently available: "offthe-shelf" and custom-made devices. Off-the-shelf devices consist of a standard multi-branch (4 branches) endograft. These have been demonstrated to be anatomically suitable for use in $88 \%$ of patients with aneurysmal disease [3]. Excellent clinical results have been reported in elective [4] and emergency [5] settings. On the other hand, different manufacturers have developed a customization programme to overcome the known limits of the "off-the-shelf" devices, for example anatomy unfit for standard devices, patients that require additional branches or those in the retrograde direction, and patients whose aortic diameter is outside of the standard sizes [6]. Jotec GmbH (Hechingen, Germany EU) E-xtra design programme was launched back in 2011, but to date, only a few studies on the utility of fenestrated or multi-branch devices [7-9] have been reported and no long-term results are available. For these reasons the aim of this study is to report the long-term follow-up of a large cohort of patients treated with a Jotec custom-made endograft for thoraco-abdominal aneurysms and type B dissection and to identify technical and clinical factors that predict the need for re-intervention.

\section{Materials and Methods}

A retrospective analysis of our electronic database and clinical records of all patients treated with an E-xtra design engineering endograft from Jotec $\mathrm{GmbH}$ (Hechingen, Germany EU) was performed. From the time the device became available in January 2011 till January 2017, we treated 49 patients $(71.3 \pm 9.5$ years old; 15 females). Demographic and clinical characteristics of the study are summarised in Table 1.

Indication for treatment was a Crawford type 4 aneurysm in 25 patients (51\%), type 3 in 13 patients (26.5\%), type 2 in 4 patients (8.1\%), type 1 in 2 patients (4\%) and a false lumen aneurysmal dilatation of a chronic dissection; in particular Stanford type B in 3 cases (6.1\%) and a residual dissection, after a type A surgical repair in 2 cases (4\%). All patients had been discussed at our multidisciplinary team meeting attended by interventional radiologists and cardiothoracic surgeons. The cases were all deemed unfit for open surgery due to either prior surgery precluding further repair or their co-morbidities making surgical risk unacceptably high. Mean aneurysm diameter was $58.7 \pm 8.4 \mathrm{~mm}$ (range 50-78). Indication for the treatment of aneurysms $<6 \mathrm{~cm}$ was a rapid sac enlargement ( $>5 \mathrm{~mm}$ at 6-month follow-up) or a morphology known to be at high risk of rupture (e.g. eccentric dilation).

All procedural details were recorded (procedural mean fluoroscopy time, mean contrast media volume administered during procedure and mean intervention duration).

\section{Device Planning}

All planning was performed by the same operator with more than 20 years of experience in advanced aortic procedures. Custom-made devices were planned on the basis of CT angiography (CTA) measurements taken within 3 months of the fenestrated-EVAR (f-EVAR) or branchedEVAR (b-EVAR) procedures [10]. Fenestration devices were used in all those cases in whom the endograft diameter could satisfactorily appose the aortic wall at the level of the visceral vessels. External branched devices were employed in cases where the aorta was aneurysmal at the level of the visceral vessels. If this segment was too dilated for a fenestrated device, but also not dilated enough to allow for the deployment of external branches, a device with internal branches was utilised. The orientation of the branch, either antegrade or retrograde, was chosen in order to conform to the native vessel anatomy. Retrograde branches were reserved for cases of complex aortic morphology or in patients in which technical constraints did not allow antegrade orientation, e.g. two closely positioned branches. A suitable proximal and distal landing zone, 
Table 1 Study population demographic and clinical characteristics

\begin{tabular}{ll}
\hline Population characteristics & $(N=49)$ \\
\hline Male/female & $34 / 15$ \\
Age (years) & $71.3 \pm 9.5$ \\
Risk factors & \\
Hypertension & $36(73.4 \%)$ \\
Diabetes & $7(14.2 \%)$ \\
Dyslipidaemia & $16(32.6 \%)$ \\
Renal insufficiency & $10(20.4 \%)$ \\
Ex-smoker & $22(44.9 \%) ;$ \\
Never smoker & $12(24.5 \%) ;$ \\
Current smoker & $15(30.6 \%)$ \\
At least one previous aortic intervention & $21(42.8 \%)$ \\
Elephant trunk & 7 \\
Great vessel transposition & 1 \\
Femoro-femoral bypass & 1 \\
Aortic-bifemoral bypass & 4 \\
Mechanical aortic valve & 1 \\
TEVAR & 7 \\
Descendant aortic surgery & 1 \\
EVAR & $38.7 \pm 8.4(50-78)$ \\
Ascendant aortic surgery & \\
Coronary bypass & 13 \\
Heart transplant & 5 \\
Indication for treatment & 1 \\
Crawford 4 & 25 \\
Crawford 3 & 5 \\
Crawford 2 & 5 \\
Crawford 1 & 1 \\
Dissection & \\
Mean aneurysm diameter (mm) & \\
\hline
\end{tabular}

either surgical (e.g. proximal elephant trunk or distal aortic-bifemoral bypass) or native $(>2 \mathrm{~cm})$, were employed in all cases. Completion of the fenestration was performed with balloon-expandable covered stents (Advanta V12, Maquet Holding, Germany), whereas branch completion was performed with self-expandable covered stents (Viabahn, Gore \& Associates, Flagstaff, USA). Figures 1 and 2 show two challenging cases performed accordingly to our customization policy.

\section{Procedure}

All procedures were performed in a single session in a state-of-the-art angio-suite, under general anaesthesia. Custom-made devices were deployed via a surgical cut down of the femoral artery in all cases. Endovascular fenestration was performed, according to the previously published techniques [11], in all patients with dissection in whom the dissection membrane involved the juxtavisceral aorta. Catheterization of the visceral vessel fenestration or branch and consequent stenting was performed via a surgical left femoral access in all cases that needed an antegrade approach. Spinal cerebrospinal fluid (CSF) drainage was employed only in those cases in whom an associated thoracic endovascular aortic repair (TEVAR) procedure was planned or in patients with previous aortic procedures.

\section{Follow-Up}

Follow-up was performed with CTA at 30 days, 6 months, 1 year and annually thereafter [10]. In cases in which CTA depicted suspicious findings but did not require immediate re-intervention stricter imaging surveillance was performed. In all cases that required re-intervention, it was performed within 30 days. Moreover, all CTA datasets were studied in order to identify the presence of endoleaks. Complications were classified according to the CIRSE classification system [12].

\section{Statistical Analysis}

Continuous data were described as the mean value $\pm \mathrm{SD}$, whereas non-Gaussian with median and percentiles. We took the clinical characteristics of the patients who had reinterventions on the aneurysmal sac. For re-intervention on fenestrations/branches all the technical features relating to the endovascular reconstruction like the number of stents employed, the length of the stented segment, fenestration versus branch, inner versus external branch, antegrade versus retrograde branch, the presence of interposed adjunctive stent (bridge stent) and vessel diameter were analysed. Multiple logistic regression analysis was performed to test the variables, and Kaplan-Meier analysis was performed to measure the fraction of subjects without complication for a certain amount of time after treatment. A $p$ value $<0.05$ was regarded to indicate a statistically significant association. All $p$ values were calculated using a two-tailed significance level. Statistical analysis was performed with the SPSS 13.0 statistical package (SPSS Inc, Chicago, IL). Graphics were plotted with MedCalc 15.0 software (MedCalc, Mariakerke, Belgium).

\section{Definition}

Technical success was defined as endograft deployment and catheterization success of fenestrations and/or branches.

With regard to fenestration/branch patency, primary patency is defined as an uninterrupted patency without adjunctive interventions. Primary-assisted patency defines the patency of a fenestration/branch after re-intervention 

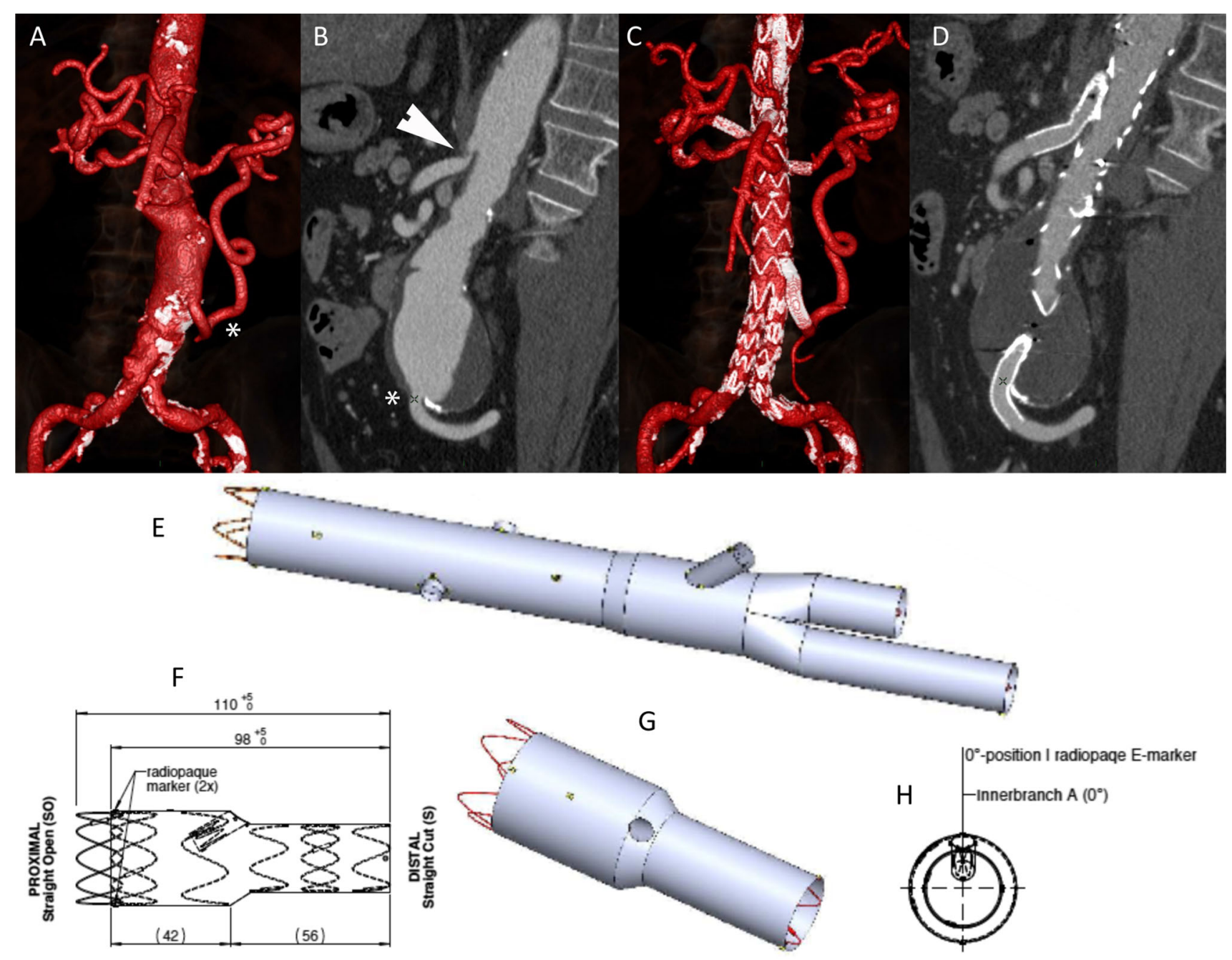

Fig. 1 Clinical case of a 67-year-old male patient with a 52-mm Crawford type 4 aneurysm. A Pre-procedural CT angiography (CTA) volume rendering (VR) reconstruction shows that the aneurysm involved the splanchnic vessels and depicts a hypertrophied inferior mesenteric artery (IMA; asterisk). B Oblique multi-planar reconstruction (MPR) confirms an occluded coeliac trunk, a pre-occlusive stenosis of the superior mesenteric artery (SMA; arrowhead) and a

for a restenosis/disconnection, but no occlusion. Secondary patency defines as initially patent but then occluded side branch, which was reopened successfully.

Based on CTA measurements (maximum diameter) aneurysm sac behaviour was classified as follows: reduced (reduction greater than $5 \mathrm{~mm}$ ), stable and enlarging (increase greater than $5 \mathrm{~mm}$ ).

\section{Results}

Endograft deployment was achieved in all cases. The custom-made endografts comprised: fenestrated $(n=21$; $42.8 \%)$, multi-branch $(n=24 ; 49 \%)$, and mixed $(n=4$; hypertrophied inferior mesenteric artery (IMA; asterisk) with an ostial stenosis. C Four-year CTA follow-up demonstrates patency of the endograft, all branches and fenestrations. E, H Endograft project was performed by selecting a proximal shorter segment with an inner branch for the SMA $(\mathbf{F}-\mathbf{H})$ and a second distal bifurcated custom device with two fenestrations for the renal arteries and an external branch for the IMA (E)

$8.1 \%)$. In three patients with dissection endovascular fenestration of the dissecting membrane was performed prior to endograft deployment.

Catheterization of fenestration/branch was successful in 152/156 (97.4\%) vessels. Details of the involved vessels and the types of reconstruction are summarized in Table 2.

In four patients a renal artery could not be catheterized. This was solved with chimney technique in one case; in two cases, the renal fenestrations were left unstented, but no late endoleak occurred. In the last case the left renal artery was impossible to catheterize. The corresponding branch was embolized with an Amplatzer vascular plug (St Jude Medical, Zaventem, Belgium). Of these four patients two experienced a significant renal infarction but did not 


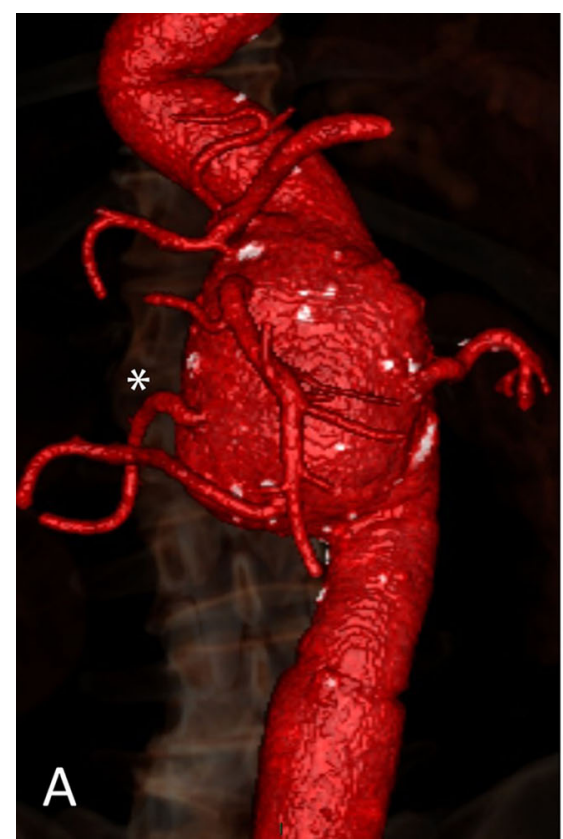

B
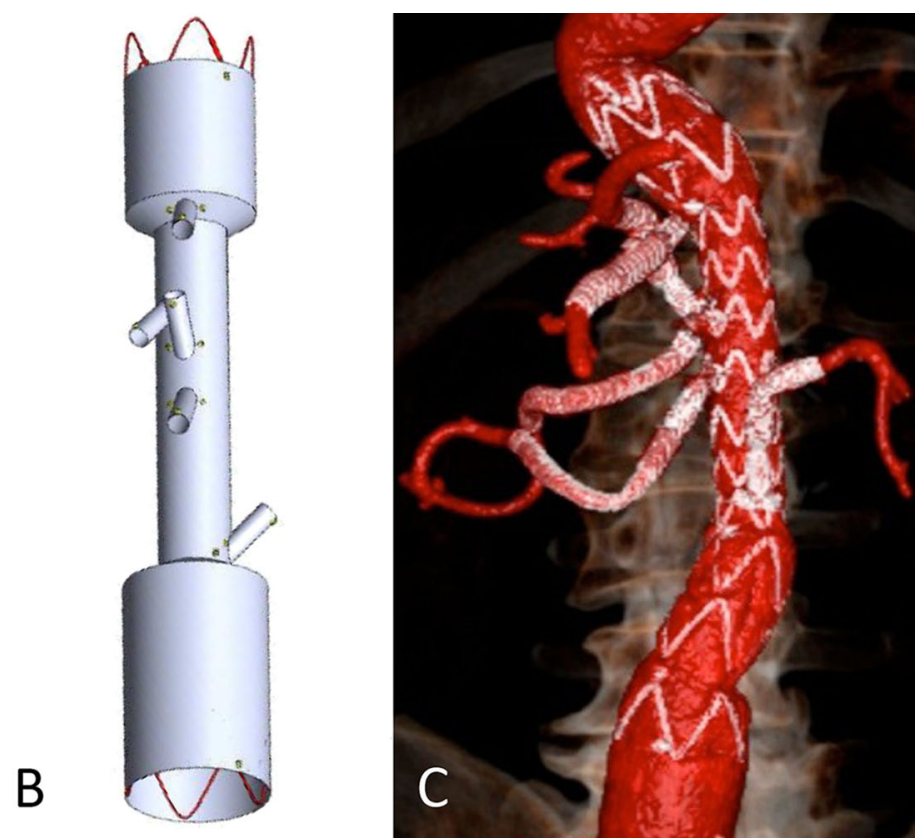

Fig. 2 Clinical case of a 70-year-old male patient with a $78-\mathrm{mm}$ Crawford type 3 aneurysm after previous surgical repair of the descendent aorta and of the abdominal aorta. A Pre-procedural CT angiography (CTA) volume rendering (VR) reconstruction shows that the aneurysm involves all the splanchnic vessels origins and depicts a right accessory renal artery (asterisk). B Selected endograft project was a single segment with dog bone shape, utilized in order to

require dialysis, but just a mild increase in creatinine level $(1.4 \mathrm{mg} / \mathrm{dL})$.

Procedural mean fluoroscopy time was $79 \pm 34 \mathrm{~min}$; mean contrast media volume administered during the procedures was $212 \pm 93 \mathrm{ml}$; mean intervention duration was $330 \pm 120 \mathrm{~min}$. Associated surgical interventions were a carotid-subclavian bypass, one femoro-femoral bypass and three common femoral endarterectomies at the access site. Spinal CSF drainage was used in eleven cases. facilitate external branch opening, with three antegrade external branches (coeliac trunk, SMA and right renal artery) and two retrograde external branches (right accessory renal artery and left renal artery) performed to facilitate catheterization due to the particular steep angles. Ceailing was performed over proximal and distal surgical neck. C Five-year CTA follow-up demonstrates the patency of the endograft and all antegrade and retrograde branches

Intra-procedural embolization of aortic side branches was performed prior to endograft deployment in 14 patients (28.5\%). Different embolic materials were employed (plug/coils) according to the vessels dimension and morphology. 5 inferior mesenteric arteries, 4 hypogastric arteries, 3 accessory renal arteries, 1 left gastric artery arising directly from the aorta and 1 common iliac were embolized. In each case the vessel embolized potentially would have been responsible for an endoleak.
Table 2 Summary of typology and site of fenestrations and branches reconstruction

\begin{tabular}{ll}
\hline Technical success fenestrations/branches catheterization & $97.4 \%(152 / 156)$ \\
\hline Vessels & \\
Coeliac trunk & 28 \\
Superior mesenteric artery & 36 \\
Right renal artery & $45^{*}$ \\
Left renal artery & $47^{*}$ \\
Accessory renal artery & 3 \\
Inferior mesenteric artery & 1 \\
Fenestrations & $N=55$ \\
Branches & $N=97$ \\
Branches type: internal versus external & 22 versus 75 \\
Branches orientation: antegrade versus retrograde & 77 versus 20 \\
\hline
\end{tabular}

*Two right renal and two left renal vessels were not catheterized 
Peri-procedural (30-day) complications were observed in ten patients $(20.4 \%)$. Three patients experienced paraplegia: one permanent (grade 5) and two transient solved by spinal CSF drainage (grade 3). Three cases of haemorrhage, in particular 1 renal and 2 retroperitoneal, were all successfully treated with embolization; one mild pancreatitis (grade 3) was conservatively managed; 1 aortic perforation was treated with an adjunctive distal aortic cuff (grade 3 ).

One iliac artery rupture was surgically managed (grade 2); 2 cases with cerebrovascular accident occurred (CVA) (grade 5).

Thirty-day mortality was $10.2 \%(n=5)$; the cause of death included two patients with multi-organ failure, two CVA and one myocardial infarction. 180-day mortality was $14.3 \%(n=7) ; 2$ additional deaths were not related to aortic pathology.

Mean follow-up was $23.6 \pm 20.9$ months (range 1-80). No patients needed surgical explant or experienced kidney function impairment related to contrast media administration. CTA follow-up showed sac shrinkage $(n=12)$ or a stable diameter $(n=24)$ in $36 / 49(73.4 \%)$; in the remaining cases $(9 / 49 ; 18.3 \%)$, sac enlargement was appreciable. All these nine patients underwent re-intervention.

Re-intervention was performed in sixteen patients (34.67\%). Three patients had a branch/stent disconnection $(n=3)$ treated with endovascular relining (Fig. 3). In 3 patients a branch/stent occlusion was managed endovascularly, with no clinical sequelae. One iliac limb occlusion was treated with thrombolysis and stenting. There were ten interventions for endoleak. Endoleak rate was $34.6 \%$ $(n=17)$, in particular twelve type II, one type Ia, one type Ib and three type III endoleak; of these seventeen patients, only nine required further treatment. Table 3 summarizes indication and re-intervention type.

Kaplan-Meier curve results for primary, primary-assisted and secondary patency of fenestrations/branches are shown in Fig. 4. In particular at 80 months the reported patency percentage was 90,96 and $100 \%$, respectively.

Logistic regression results demonstrated that the need for re-intervention on the aneurysm sac was related to dissection, previous aortic surgery and intra-procedural embolization of aortic side branches, whereas the requirement for re-intervention on fenestrations/branches was increased in patients with branches especially external branches. Table 4 summarizes the results of the logistic regression analysis.

\section{Discussion}

The results of this study report the clinical outcome of a large series of patients treated for thoraco-abdominal aneurysm and false lumen aneurismal degeneration in type B chronic dissection with a Jotec custom-made endograft.
This series confirms how accurate pre-procedural planning could lead to technical success in all cases, thus increasing the number of patients that could benefit from such endovascular treatment as an alternative to open surgery. Catheterization of a renal fenestration or branch was unsuccessful in only four cases $(2.6 \%)$. Despite failed catheterization, none of these patients required further reintervention or dialysis. The reported high success rate for catheterization of fenestrations/branches is in line with that reported with other multi-branch [13-15] and fenestrated [16-18] devices. Our peri-procedural complication rate and 30-day mortality are broadly comparable with the available literature [14, 19], but it should be noted that most of our patients $(42.8 \%)$ had previously undergone an aortic surgical/endovascular intervention. Additionally, nearly $40 \%$ of the patients were treated for a Crawford type I-III thoraco-abdominal aneurysm, which is associated with higher morbidity and mortality [20-22].

Long-term follow-up demonstrates that customized f-EVAR and b-EVAR procedures were able to avoid progression of aortic disease in nearly $75 \%$ of patients, as confirmed by sequential CTA diameter measurements that depicted a stable or shrinking aneurysm sac. Moreover, no patient throughout the entire follow-up required surgical explantation. This was possible thanks to strict surveillance applied to all patients treated with f-EVAR or b-EVAR in our centre. All patients shown to have sac reperfusion (endoleak) were shifted towards shorter imaging follow-up (6 months) in the case of stable aneurysmal sac diameter or towards re-intervention in cases of an enlarging aneurysm sac. Moreover, imaging surveillance follow-up was of paramount importance to identify the cause of sac reperfusion, aiding in the selection of the best treatment option to correct it. Classical type II endoleaks are easily identified and treated; however, the instability of fenestration/ branch is more difficult to diagnose. The latter condition should be suspected in all sac reperfusions occurring around a fenestration/branch not in connection with an aortic side branches, even in the absence of frank stent disconnection. Relining of the fenestrations/branches resolved the problem in all cases. By employing this policy we were able to report an 80-month fenestration/branch secondary patency of $100 \%$, results that are slightly superior compared to those reported in the literature for both f-EVAR [14, 23-27] and b-EVAR [14, 24] series.

Multivariate analysis identified that predictors for reintervention on the aneurysmal sac were patients with dissection, previous aortic surgery and the need for intraprocedural embolization of an aortic side branch. Studying these results, it is not surprising that dissection and previous aortic surgery are statistically associated with re-intervention. In fact, these two clinical variables identify patients who are more fragile, since they are affected by a 

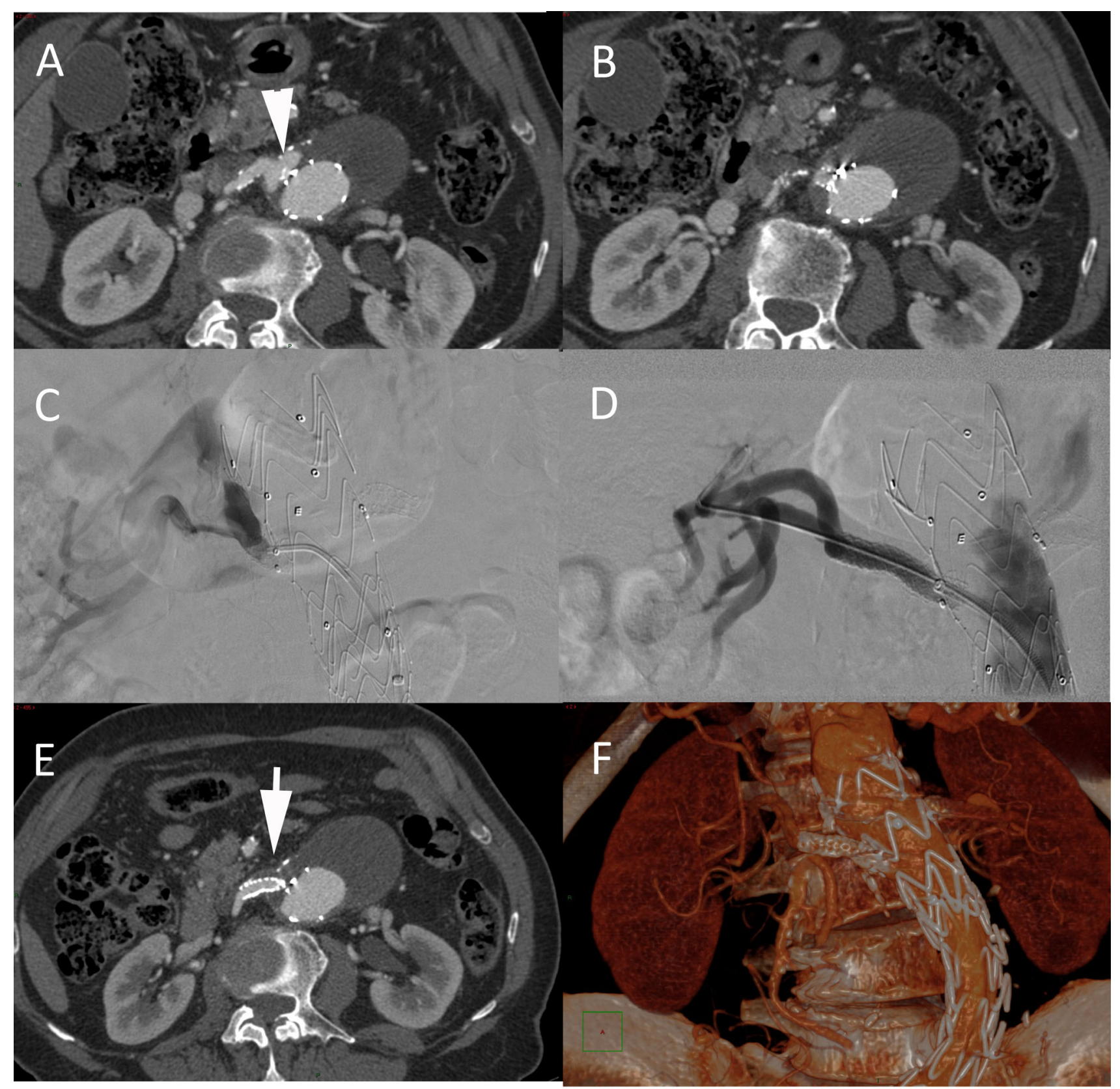

Fig. 3 Clinical case of a 69 -year-old male patient with a $70-\mathrm{mm}$ Crawford type 4 aneurysm, with the disconnection of the renal fenestration from the right renal artery. A, B Pre-procedural axial CT angiography (CTA) shows disconnection of the right renal stent from the renal artery with concomitant type III endoleak (arrowhead). C Selective digital subtraction angiography (DSA) confirms stent

rapidly evolving disease. Regarding the intra-procedural embolization of aortic side branches, no straightforward explanation is possible. In fact, none of the re-interventions was due to reperfusion of previously embolized vessels. Likely, the statistical association is due to the fact that the aneurysmal degeneration was more advanced including more aortic side branches. Finally, multivariate results demonstrated as predictors for re-intervention in disconnection and type III endoleak. D Relining of the fenestration was then performed via the groin with a longer balloon-expandable covered stent in order to restore connection between the fenestration and the right renal artery. E, F Two-year axial CTA (E) and volume rendering (VR) reconstruction $(\mathrm{F})$ follow-up demonstrate patency of the right renal fenestration with endoleak resolution (arrow)

fenestrations/branches: branch usage versus fenestration, external branch versus internal branch. Again, keeping in mind the policy of device customization that we used, branch usage and external branch were reserved for more advanced disease (e.g. Crawford type I-III aneurysm and dissection), those in which both fenestration and inner branch were not feasible. Moreover, multivariate analysis suggests that re-intervention on visceral vessels was not 
Table 3 Summary of indication and re-interventions typology

\begin{tabular}{lll}
\hline Indication for re-intervention & Type of re-intervention & $N=$ \\
\hline Branch/stent disconnection & Relining & 3 \\
Branch/stent occlusion & Fibrinolysis & 3 \\
Iliac limb occlusion & Thrombolysis and stenting & 1 \\
Endoleak type Ia & TEVAR & 1 \\
Endoleak type IA & TEVAR & 1 \\
Endoleak type IB & Sac embolization and iliac extension & 1 \\
Endoleak type II & Collateral embolization & 3 \\
Endoleak type III & Relining & 4 \\
Endoleak type III & Relining & 3 \\
\hline
\end{tabular}

Fig. 4 Primary patency (green line), primary-assisted patency (blue dotted line) and secondary patency (red dotted line) Kaplan-Meier curves

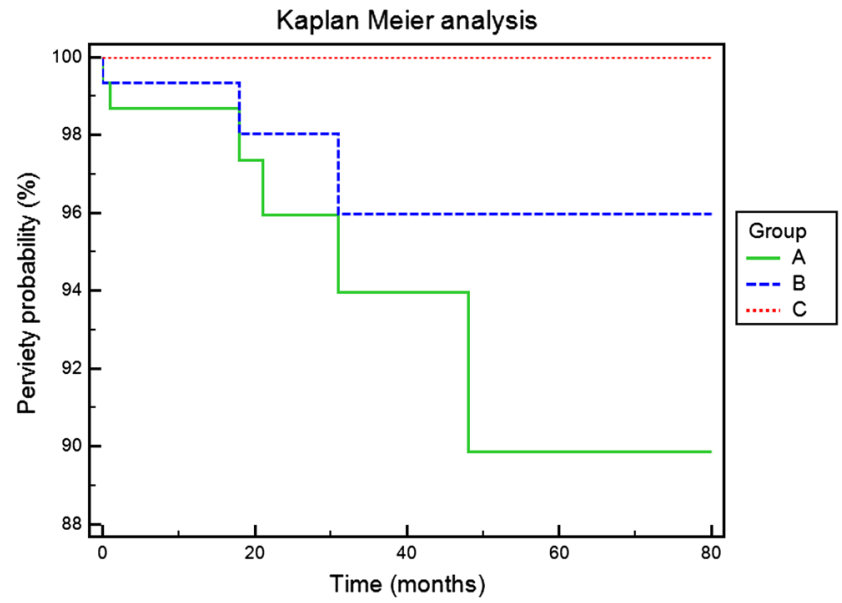

the controversial literature evidence on snorkel parallel graft and on branched devices that, respectively, advocate how steep angulation [28, 29] and certain anatomy could be unfit for endovascular repair [30].

Study limitations include the retrospective nature of the study and the highly selected patients for this alternative

Table 4 Multiple logistic regression analysis for re-interventions on aneurismatic sac and fenestrations/branches

\begin{tabular}{|c|c|c|c|c|c|}
\hline Variables & Coefficient & SE & $r_{\text {partial }}$ & $t$ & $p$ value \\
\hline \multicolumn{6}{|c|}{ Multiple logistic regression analysis for re-intervention on aneurismatic SAC } \\
\hline Dissection & 0.3969 & 0.1864 & 0.3289 & 2.284 & $0.039 *$ \\
\hline Previous aortic surgery & -0.3744 & 0.1455 & -0.3652 & -2.573 & $0.0136^{*}$ \\
\hline Intra-procedural embolization of aortic collaterals & 0.2895 & 0.1268 & 0.3289 & 2.284 & $0.0274 *$ \\
\hline \multicolumn{6}{|c|}{ Multiple logistic regression analysis for re-intervention on fenestrations/branches } \\
\hline Branch & 0.2859 & 0.123 & 0.1902 & 2.324 & $0.0215^{*}$ \\
\hline Bridge & -0.09234 & 0.07017 & -0.109 & -1.316 & 0.1903 \\
\hline Diameter & -0.005081 & 0.01309 & -0.03234 & -0.388 & 0.6984 \\
\hline Internal_external & -0.1293 & 0.04792 & -0.2193 & -2.698 & $0.0078^{*}$ \\
\hline Length & -0.0005692 & 0.0007643 & -0.06194 & -0.745 & 0.4577 \\
\hline Stent_number & 0.08215 & 0.0601 & 0.1132 & 1.367 & 0.1738 \\
\hline Orientation & -0.007093 & 0.05285 & -0.01118 & -0.134 & 0.8934 \\
\hline
\end{tabular}


endovascular treatment. In fact, all enrolled patients suffered from multiple comorbidities and underwent multiple previous aortic interventions, thus limiting the strength of the identification of prognostic factors for re-intervention on the aneurysmal sac.

In conclusion, our study reports the first long-term result of the Jotec customization platform that is comparable to previously reported similar devices, with an acceptable complication and re-intervention rate. According to the customization policy we employed, fenestrations and inner branches should be preferred whenever possible because they were associated with a lower re-intervention rate. Retrograde branches should be considered as a safe alternative in all cases not fit for antegrade branches.

\section{Compliance with Ethical Standards}

Conflict of interest All authors declare that they have no conflict of interests.

Ethical Approval All procedures performed in studies involving human participants were in accordance with the ethical standards of the institutional and/or national research committee and with the 1964 Declaration of Helsinki and its later amendments or comparable ethical standards.

Informed Consent Informed consent for the procedure for anonymized publication of this series of patients was obtained from all individual participants included in the study.

\section{References}

1. Khoynezhad A, Toluie S, Al-Atassi T. Treatment of the chronic type B aortic dissection: the pro-endovascular argument. Semin Thorac Cardiovasc Surg. 2017;29(2):131-6.

2. Rosenblum JM, Chen EP. Thoracoabdominal aortic aneurysm repair: open, endovascular, or hybrid? Gen Thorac Cardiovasc Surg. 2017.https://doi.org/10.1007/s11748-017-0820-y

3. Sweet MP, Hiramoto JS, Park KH, et al. A standardized multibranched thoracoabdominal stent-graft for endovascular aneurysm repair. J Endovasc Ther. 2009;16(3):359-64.

4. Bosiers MJ, Bisdas T, Donas KP, et al. Early experience with the first commercially available off-the-shelf multibranched endograft (t-branch) in the treatment of thoracoabdominal aortic aneurysms. J Endovasc Ther. 2013;20(6):719-25.

5. Silingardi R, Gennai S, Leone N et al. Standard "off-the-shelf" multibranched thoracoabdominal endograft in urgent and elective patients with single and staged procedures in a multicenter experience. J Vasc Surg. 2018;67(4):1005-1016.https://doi.org/ 10.1016/j.jvs.2017.08.068

6. Nordon IM, Hinchliffe RJ, Manning B, et al. Toward an "off-theshelf" fenestrated endograft for management of short-necked abdominal aortic aneurysms: an analysis of current graft morphological diversity. J Endovasc Ther. 2010;17(1):78-85.

7. Ricci C, Ceccherini C, Cini M, et al. Juxtarenal aortic aneurysms: preliminary experience with fenestrated E-vita abdominal stentgraft. J Cardiovasc Surg. 2013;54(4):491-7.

8. Kinstner C, Teufelsbauer $\mathrm{H}$, Neumayer $\mathrm{C}$, et al. Endovascular repair of thoracoabdominal aortic aneurysms with a novel multibranch stent-graft design: preliminary experience. J Cardiovasc Surg. 2014;55(4):543-50.

9. Van den Eynde W, Van Breussegem A, Joos B, et al. Endovascular repair of a type 1a endoleak after Ch-EVAR with a b-EVAR. Cardiovasc Interv Radiol. 2016;39(9):1361-3.

10. Expert Panels on Vascular I, Interventional R, Bonci G et al (2017) ACR appropriateness criteria $[(\mathrm{R})]$ thoracic aorta interventional planning and follow-up. J Am Coll Radiol JACR, 14(11S):S570-S583.

11. Ricci C, Ceccherini C, Leonini S, et al. JAG tearing technique with radiofrequency guide wire for aortic fenestration in thoracic endovascular aneurysm repair. Cardiovasc Interv Radiol. 2012;35(1):176-9.

12. Filippiadis DK, Binkert C, Pellerin O, et al. Cirse quality assurance document and standards for classification of complications: the cirse classification system. Cardiovasc Interv Radiol. 2017;40(8):1141-6.

13. Martin-Gonzalez T, Pincon C, Maurel B, et al. Renal outcomes following fenestrated and branched endografting. Eur $\mathrm{J}$ Vasc Endovasc Surg. 2015;50(4):420-30.

14. Eagleton MJ, Follansbee M, Wolski K, et al. Fenestrated and branched endovascular aneurysm repair outcomes for type II and III thoracoabdominal aortic aneurysms. J Vasc Surg. 2016;63(4):930-42.

15. Fernandez CC, Sobel JD, Gasper WJ, et al. Standard off-the-shelf versus custom-made multibranched thoracoabdominal aortic stent grafts. J Vasc Surg. 2016;63(5):1208-15.

16. Mastracci TM, Eagleton MJ, Kuramochi Y, et al. Twelve-year results of fenestrated endografts for juxtarenal and group IV thoracoabdominal aneurysms. J Vasc Surg. 2015;61(2):355-64.

17. Farber MA, Eagleton MJ, Mastracci TM, et al. Results from multiple prospective single-center clinical trials of the off-theshelf p-branch fenestrated stent graft. J Vasc Surg. 2017;66(4):982-90.

18. Shahverdyan R, Gray D, Gawenda M, et al. Single centre results of total endovascular repair of complex aortic aneurysms with custom made anaconda fenestrated stent grafts. Eur J Vasc Endovasc Surg. 2016;52(4):500-8.

19. Hu Z, Li Y, Peng R, et al. Multibranched stent-grafts for the treatment of thoracoabdominal aortic aneurysms: a systematic review and meta-analysis. J Endovasc Ther. 2016;23(4):626-33.

20. Sandmann W, Grabitz K, Torsello G, et al. Surgical treatment of thoraco-abdominal aneurysm. Indications and results. Der Chirurg; Zeitschrift fur alle Gebiete der operativen Medizen. 1995;66(9):845-56.

21. Coselli JS, LeMaire SA, Preventza O, et al. Outcomes of 3309 thoracoabdominal aortic aneurysm repairs. J Thorac Cardiovasc Surg. 2016;151(5):1323-37.

22. Guillou M, Bianchini A, Hertault A, et al. A new score to predict post operative complications after endovascular treatment of thoraco abdominal aortic aneurysms. Acta Chir Belg. 2014;114(4):250-5.

23. Halak M, Goodman MA, Baker SR. The fate of target visceral vessels after fenestrated endovascular aortic repair-general considerations and mid-term results. Eur J Vasc Endovasc Surg. 2006;32(2):124-8.

24. Reyes A, Donas KP, Pitoulias G, et al. Complementary role of fenestrated/branched endografting and the chimney technique in the treatment of pararenal aneurysms after open abdominal aortic repair. J Endovasc Ther. 2016;23(4):599-605.

25. Grimme FA, Zeebregts CJ, Verhoeven EL, et al. Visceral stent patency in fenestrated stent grafting for abdominal aortic aneurysm repair. J Vasc Surg. 2014;59(2):298-306.

26. Di X, Ye W, Liu CW, et al. Fenestrated endovascular repair for pararenal abdominal aortic aneurysms: a systematic review and meta-analysis. Ann Vasc Surg. 2013;27(8):1190-200. 
27. Verhoeven EL, Vourliotakis G, Bos WT, et al. Fenestrated stent grafting for short-necked and juxtarenal abdominal aortic aneurysm: an 8-year single-centre experience. Eur J Vasc Endovasc Surg. 2010;39(5):529-36.

28. Caradu C, Berard X, Midy D, et al. Influence of anatomic angulations in chimney and fenestrated endovascular aneurysm repair. Ann Vasc Surg. 2017;43:104-14.
29. Ullery BW, Suh GY, Kim JJ, et al. Dynamic geometric analysis of the renal arteries and aorta following complex endovascular aneurysm repair. Ann Vasc Surg. 2017;43:85-95.

30. Mendes BC, Oderich GS, Reis de Souza L, et al. Implications of renal artery anatomy for endovascular repair using fenestrated, branched, or parallel stent graft techniques. J Vasc Surg. 2016;63(5):1163-1169 e1161. 